\title{
OSCILLATION CRITERIA FOR SECOND-ORDER LINEAR DIFFERENTIAL EQUATIONS $\left({ }^{1}\right)$
}

\author{
BY \\ ZEEV NEHARI
}

1. Introduction. In this paper we shall be concerned with the differential equation

$$
y^{\prime \prime}+p(x) y=0,
$$

where $p(x)$ is a continuous positive function for $0<x<\infty$. Equation (1) is said to be nonoscillatory in $(a, \infty)$ if no solution of (1) vanishes more than once in this interval. Because of the Sturm separation theorem, this is equivalent to the existence of a solution which does not vanish at all in $(a, \infty)$. The equation will be called nonoscillatory-without the interval being mentioned -if there exists a number $a$ such that (1) is nonoscillatory in $(a, \infty)$. The equation (1) will be said to be oscillatory, if one (and therefore all) of its solutions have an infinite number of zeros for $x>0$.

Our main concern will be to obtain oscillation criteria (or nonoscillation criteria) for the equation (1), i.e. conditions on the function $p(x)$ from which conclusions may be drawn as to the oscillatory, or nonoscillatory, character of (1). There exists an extensive literature on this subject [2-12, to name just a few], which goes back to a classical paper by Kneser [5]. Considerable progress was made in a relatively recent paper by Hille [4], who recognized the relevance of the expression

$$
g(x)=x \int_{x}^{\infty} p(x) d x
$$

for the discussion of the oscillation problem (here it is, of course, assumed that the integral exists; if it does not, $(1)$ is known to be oscillatory $[2 ; 6 ; 11])$. If the.quantities $g_{*}$ and $g^{*}$ are defined as

$$
g_{*}=\liminf _{x \rightarrow \infty} g(x), \quad g^{*}=\limsup _{x \rightarrow \infty} g(x),
$$

Hille showed that $g_{*} \leqq 1 / 4, g^{*} \leqq 1$ if (1) is nonoscillatory, and $g^{*} \geqq 1 / 4$ if the equation is oscillatory, where all the inequalities are sharp.

In the present paper we shall develop more general oscillation criteria which will contain Hille's criteria as special cases. The basic idea used is the fact that there exists an intimate connection between the oscillation problem

Received by the editors July 5, 1956.

(1) This research was supported by the United States Air Force Office of Scientific Research. 
for the equation (1) and the eigenvalue problem for the equation $u^{\prime \prime}+\lambda p(x) u$ $=0$ with suitable boundary conditions. While this connection is very simple indeed, its bearing upon the oscillation problem does not seem to have been noticed, and we state it therefore as a separate theorem.

THEOREM I. If $\lambda$ denotes the lowest eigenvalue of the system

$$
u^{\prime \prime}+\lambda p(x)=0, \quad u(a)=u^{\prime}(b)=0,
$$$$
a<b,
$$

then (1) is nonoscillatory in $(a, \infty)$ if, and only if, $\wedge>1$ for all $b$ such that $b>a$.

If (1) is nonoscillatory in $(a, \infty)$ and $y(x)$ is a solution of (1) such that $y(a)=0, y^{\prime}(a)>0$ then, as pointed out by Hille, $y^{\prime}(x)>0$ for all $x \geqq a$. Using (1) and (4), we find

$$
0<u(b) y^{\prime}(b)=-\int_{a}^{b}\left(y u^{\prime \prime}-u y^{\prime \prime}\right) d x=(\lambda-1) \int_{a}^{b} p u y d x .
$$

Since $p, u, y$ are non-negative in $(a, b)$, it follows that $\lambda>1$. The same identity also shows, conversely, that $y^{\prime}(b)$ must be positive if $\lambda>1$ and $y \geqq 0 . y(x)$ is certainly positive for values of $x$ which are slightly larger than $a$. If we let $b$ grow, starting from values which are near $a, y^{\prime}(b)$ will remain positive as long as $y$ does not become negative. Since, however, $y$ cannot become negative unless $y^{\prime}$ has become negative at a lower value of $x$, this cannot happen. Hence (1) is nonoscillatory in $(a, \infty)$ if $\lambda>1$ for all $b>a$.

In view of Theorem $I$ it is natural to subdivide the class of equations (1) according to the following definitions.

(a) The equation (1) is said to be strongly oscillatory if the equation

$$
y^{\prime \prime}+\lambda p(x) y=0
$$

is oscillatory for all positive values of $\lambda$.

(b) (1) is strongly nonoscillatory if $\left(4^{\prime}\right)$ is nonoscillatory for all positive $\lambda$.

(c) (1) is conditionally oscillatory if $\left(4^{\prime}\right)$ is oscillatory for some positive $\lambda$ and nonoscillatory for some other $\lambda>0$.

From the Sturm comparison theorem it follows that in the case (c) there must exist a positive number $\mu$ such that (1) is oscillatory for $\lambda>\mu$ and nonoscillatory for $\lambda<\mu$. This number $\mu$ will be called the oscillation constant of the equation (1). The cases (a) and (b) may also be characterized by the conditions $\mu=0$ and $\mu=\infty$, respectively.

Cases (a) and (b) can be easily disposed of by means of the results of Hille quoted further above.

THEOREM II. Equation (1) is strongly oscillatory if, and only if,

$$
\limsup _{x \rightarrow \infty} x \int_{x}^{\infty} p(x) d x=\infty \text {. }
$$

Equation (1) is strongly nonoscillatory if, and only if, 


$$
\lim _{x \rightarrow \infty} x \int_{x}^{\infty} p(x) d x=0 .
$$

Indeed, if (1) is strongly-oscillatory and $g^{*}$ is the quantity defined in (3), we must have $\lambda g^{*} \geqq 1 / 4$ for all positive $\lambda$, which shows that $g^{*}=\infty$. Conversely, if $g^{*}=\infty$ then $\lambda g^{*}>1$ for all positive $\lambda$ and (2) is oscillatory for all such $\lambda$. To verify the second half of Theorem II, we observe that if (1) is strongly nonoscillatory, the inequality $\lambda g^{*} \leqq 1$ must hold for all positive $\lambda$. Hence, $g^{*}=0$. Conversely, if $g^{*}=0$, we have trivially $\lambda g^{*}<1 / 4$, and everything is proved.

We are thus left with case (c), i.e., with equations (1) which oscillate conditionally. It is clear that we cannot hope to obtain a simple necessary and sufficient criterion for the nonoscillation of such equations. As Theorem I shows, this would essentially amount to devising a method for finding the lowest eigenvalue of the differential system (4) by means of elementary operations.

In $\S 2$, we shall show by a number of examples how Theorem I can be used to obtain necessary conditions for nonoscillation which go considerably further than those found in the literature. The proofs will be based on the minimum property of the lowest eigenvalue of the system (4). By its very nature, this property can yield only necessary, but not sufficient, conditions for nonoscillation. In order to obtain sufficient conditions, it seems to be necessary to use procedures which involve some kind of comparison of the given equation with one whose oscillatory character is known. The theorems of $\S 4$ will be based on comparison procedures of this type. By a proper utilization of the minimum property of the first eigenvalue, the method used may be made to yield sufficient conditions both for oscillation and nonoscillation. The comparison principle employed is also useful in the treatment of other problems related to the equation (1), and we therefore state and prove it as a separate comparison theorem in $\$ 3$.

In $\S 5$, we shall derive formulas for the asymptotic estimation of the number of zeros of the solutions of certain classes of oscillatory equations (1). These formulas generalize a result first proved by Wiman [10] and rediscovered by Hartman and Wintner [3]. The proofs are again based on the consideration of a suitable related eigenvalue problem.

2. Properties of nonoscillatory equations. If $\lambda$ is the lowest eigenvalue of the differential system (4), then

$$
\lambda \int_{a}^{b} p(x) y^{2}(x) d x \leqq \int_{a}^{b} y^{\prime 2}(x) d x
$$

where $y(x) \in D^{1}$ in $[a, b]$ and $\lim y^{2}(x)(x-a)^{-1} \rightarrow 0$ if $x$ approaches $a$ from the right. The proof of this well-known minimum property of the lowest eigen- 
value is an immediate consequence of the identity

$$
\begin{aligned}
0 \leqq \int_{a+\epsilon}^{b}\left(y^{\prime}-\frac{u^{\prime}}{u} y\right)^{2} d x & =\int_{a+\epsilon}^{b} y^{\prime 2} d x-2 \int_{a+\epsilon}^{b} \frac{u^{\prime}}{u} y y^{\prime} d x+\int_{a+\epsilon}^{b} \frac{u^{\prime 2}}{u^{2}} y^{2} d x \\
& =\int_{a+\epsilon}^{b} y^{\prime 2} d x+\int_{a+\epsilon}^{b} \frac{u^{\prime \prime}}{u} y^{2} d x-\left[\frac{u^{\prime}}{u} y^{2}\right]_{a+\epsilon}^{b},
\end{aligned}
$$

where $u(x)$ may be taken to be any function of class $C^{1}$ which does not vanish in $(a, b)$ and $a<a+\epsilon<b$. If we identify $u(x)$ with the first eigenfunction of the system (4) and let $\epsilon$ tend to zero, we obtain (5).

By Theorem I, $\lambda$ will be larger than unity if (1) is nonoscillatory in $(a, \infty)$. In view of (5), such equations will therefore give rise to the inequality

$$
\int_{a}^{b} p(x) y^{2}(x) d x<\int_{a}^{b} y^{\prime 2}(x) d x,
$$

where $y(x)$ is a function of class $D^{1}$ in $(a, \infty)$ for which $(x-a)^{-1} y^{2}(x) \rightarrow 0$ if $x \rightarrow a_{+}$, and $b$ is such that $b>a$.

We first illustrate the application of (6) in a very simple case. We choose a point $x_{0}$ such that $a<x_{0}<b$, and set $y(x)=(x-a)\left(x_{0}-a\right)^{-1}$ for $a \leqq x \leqq x_{0}$, and $y(x)=1$ for $x \geqq x_{0}$. This leads to

$$
\int_{x_{0}}^{b} p(x) d x \leqq \int_{a}^{b} p(x) y^{2} d x<\frac{1}{x_{0}-a} .
$$

Since $b$ may be taken arbitrarily large, this shows that $\int_{a}^{\infty} p(x) d x$ exists if (1) is nonoscillatory in $(a, \infty)[2 ; 6 ; 11]$ and that, moreover,

$$
(x-a) \int_{x}^{\infty} p(t) d t<1 .
$$

Hille's result that $g^{*} \leqq 1[4]$, where $g^{*}$ is defined by (2) and (3), follows by letting $x$ tend to $\infty$.

More generally, we set $y(x)=(x-a)^{\beta / 2}\left(x_{0}-a\right)^{-\beta / 2}$ for $a \leqq x \leqq x_{0}$ and $y(x)$ $=(x-a)^{\alpha / 2}\left(x_{0}-a\right)^{-\alpha / 2}$ for $x \geqq x_{0}$, where $\beta>1$ and $0 \leqq \alpha<1$. Inserting this in (6), we obtain

$$
\begin{aligned}
\frac{1}{\left(x_{0}-a\right)^{\beta}} & \int_{a}^{x_{0}}(x-a)^{\beta} p(x) d x+\frac{1}{\left(x_{0}-a\right)^{\alpha}} \int_{x_{0}}^{b}(x-a)^{\alpha} p(x) d x \\
& \leqq \frac{\beta^{2}}{4(\beta-1)\left(x_{0}-a\right)}+\frac{\alpha^{2}}{4(1-\alpha)\left(x_{0}-a\right)^{\alpha}}\left[\left(x_{0}-a\right)^{\alpha-1}-(b-a)^{\alpha-1}\right] .
\end{aligned}
$$

If we let $b \rightarrow \infty$, we obtain the following result.

TheOREM III. If (1) is nonoscillatory in $(a, \infty)$ and $\beta>1,0 \leqq \alpha<1$, then 


$$
\begin{aligned}
& (x-a)^{1-\beta} \int_{a}^{x}(t-a)^{\beta} p(t) d t \\
& \quad+(x-a)^{1-\alpha} \int_{x}^{\infty}(t-a)^{\alpha} p(t) d t \leqq \frac{\beta-\alpha}{4}\left[1+\frac{1}{(\beta-1)(1-\alpha)}\right] .
\end{aligned}
$$

Since both terms on the left-hand side of (7) are non-negative, this yields the separate inequalities

$$
(x-a)^{1-\beta} \int_{a}^{x}(t-a)^{\beta} p(t) d t \leqq \frac{\beta^{2}}{4(\beta-1)}, \quad \quad \beta>1,
$$

(for $\alpha=0$ ), and

$$
(x-a)^{1-\alpha} \int_{x}^{\infty}(t-a)^{\alpha} p(t) d t \leqq \frac{(2-\alpha)^{2}}{4(1-\alpha)}, \quad 0 \leqq \alpha<1,
$$

(for $\beta=2$ ). Letting $x \rightarrow \infty$, we obtain

$$
\limsup _{x \rightarrow \infty} x^{1-\beta} \int_{a}^{x} t^{\beta} p(t) d t \leqq \frac{\beta^{2}}{4(\beta-1)}
$$

and

$$
\limsup _{x \rightarrow \infty} x^{1-\alpha} \int_{x}^{\infty} t^{\alpha} p(t) d t \leqq \frac{(2-\alpha)^{2}}{4(1-\alpha)}, \quad 0 \leqq \alpha<1 .
$$

(8) also contains the fact that $x^{\alpha} p(x)(0 \leqq \alpha<1)$ is integrable in $(a, \infty)$ if $(1)$ is nonoscillatory [4].

If the quantity $\sigma(x)$ is defined by

$$
\sigma(x)=(x-a)^{1-\alpha} \int_{x}^{\infty}(t-a)^{\alpha} p(t) d t, \quad 0 \leqq \alpha<1,
$$

Theorem III may be cast into the following form.

THEOREM IIIa. If (1) is nonoscillatory in $(a, \infty), \beta>1$, and $\sigma(x)$ is defined by (10), then

$$
(x-a)^{1-\beta} \int_{a}^{x}(t-a)^{\beta-2} \sigma(t) d t \leqq \frac{1}{4}+\frac{1}{4(\beta-1)(1-\alpha)} .
$$

(11) follows from (7) by the use of the identity

$$
\begin{aligned}
\int_{a}^{x}(t-a)^{\beta} p(t) d t & =-\int_{a}^{x}(t-a)^{\beta-\alpha}\left[(t-a)^{\alpha-1} \sigma(t)\right]^{\prime} d t \\
& =-(x-a)^{\beta-1} \sigma(x)+(\beta-\alpha) \int_{a}^{x}(t-a)^{\beta-2} \sigma(t) d t
\end{aligned}
$$


and some elementary manipulation.

As an application of Theorem IIIa, we prove

TheOREM IV. If (1) is nonoscillatory, then

$$
\liminf _{x \rightarrow \infty} x^{1-\alpha} \int_{x}^{\infty} t^{\alpha} p(t) d t \leqq \frac{1}{4(1-\alpha)}, \quad 0 \leqq \alpha<1 .
$$

This bound is sharp, as shown by the nonoscillatory equation

$$
y^{\prime \prime}+\frac{y}{4 x^{2}}=0 .
$$

We choose a point $x_{0}$ such that $a<x_{0}<x$ and write (11) in the form

$$
\frac{\int_{a}^{x_{0}}(t-a)^{\beta-2} \sigma(t) d t}{(x-a)^{\beta-1}}+\frac{\int_{x_{0}}^{x}(t-a)^{\beta-2} \sigma(t) d t}{(x-a)^{\beta-1}} \leqq \frac{1}{4}+\frac{1}{4(\beta-1)(1-\alpha)} .
$$

The first term tends to zero if $x \rightarrow \infty$ and $x_{0}$ is kept fixed. If $m=\min \sigma(t)$ for $x_{0} \leqq t \leqq x$, the second term can be estimated from below by $m(\beta-1)^{-1}$. This leads to

$$
\inf _{x_{0} \leqq t<\infty} \sigma(t) \leqq \frac{\beta-1}{4}+\frac{1}{4(1-\alpha)} .
$$

The left-hand side is independent of $\beta$ and we may therefore let $\beta \rightarrow 1$. Since the resulting inequality holds for arbitrarily large values of $x_{0}$, this proves Theorem IV. It may be remarked that for $\alpha=0$, (12) reduces to the result of Hille mentioned further above.

For $\beta=2$, (11) yields

$$
\frac{1}{x-a} \int_{a}^{x} \sigma(t) d t \leqq \frac{2-\alpha}{4(1-\alpha)},
$$

which is a sharper bound than that obtainable by integrating the inequality (8). For

$$
\sigma_{0}(x)=(x-a) \int_{x}^{\infty} p(x) d x
$$

we find, in particular,

$$
\frac{1}{x-a} \int_{a}^{x} \sigma_{0}(t) d t \leqq \frac{1}{2},
$$

which should be compared with the fact, proved above, that $\sigma_{0}(x)$ itself satisfies the sharp inequality $\sigma_{0}(x)<1$. 
As a further example, we prove the following result.

If $0 \leqq p(x) \leqq m^{2}$, and (1) is nonoscillatory, in $(a, \infty)$ then there exists a universal constant $c_{0}$ such that the inequality

$$
\int_{a}^{\infty} p(x) d x \leqq c m
$$

holds for $c=c_{0}$ but not, in general, for $c=c_{0}-\epsilon, \epsilon>0$. $c_{0}$ satisfies the inequalities

$$
\frac{1}{2} \leqq c_{0}<3^{3 / 4} 2^{-1 / 2}=1.61 \ldots
$$

The lower bound for $c_{0}$ follows from the consideration of the equation (13) which is nonoscillatory in $(a, \infty), a>0$. To obtain the upper bound, we set

$$
\begin{array}{rlrl}
y(x) & =\left(\int_{a}^{x} p(t) d t\right)^{\nu}, & a \leqq x \leqq x_{0}, \nu>1 / 2, \\
y(x)=\left(\int_{a}^{x_{0}} p(t) d t\right)^{\nu}, & x_{0} \leqq x \leqq b,
\end{array}
$$

in (6). This leads to

$$
\begin{aligned}
\frac{1}{2 \nu+1}\left(\int_{a}^{x_{0}} p(t) d t\right)^{2 \nu+1}+\left(\int_{a}^{x_{0}} p(t) d t\right)^{2 \nu} \cdot \int_{x_{0}}^{b} p(t) d t \\
\quad \leqq \nu^{2} \int_{a}^{x_{0}} p^{2}\left(\int_{a}^{x} p(t) d t\right)^{2 \nu-2} d x \leqq \nu^{2} m^{2} \int_{a}^{x_{0}} p\left(\int_{a}^{x} p(t) d t\right)^{2 \nu-2} d x \\
=\frac{\nu^{2} m^{2}}{2 \nu-1}\left(\int_{a}^{x_{0}} p(t) d t\right)^{2 \nu-1}
\end{aligned}
$$

Letting $b \rightarrow \infty$ and choosing $x_{0}$ such that

$$
\int_{a}^{x_{0}} p(t) d t=\frac{2 \nu+1}{4 \nu} \int_{a}^{\infty} p(t) d t, \quad \int_{x_{0}}^{\infty} p(t) d t=\frac{2 \nu-1}{4 \nu} \int_{a}^{\infty} p(t) d t,
$$

we obtain

$$
\left(\int_{a}^{\infty} p(t) d t\right)^{2} \leqq \frac{8 \nu^{3} m^{2}}{4 \nu^{2}-1} .
$$

Setting $\nu=3^{1 / 2} / 2$, we arrive at the upper bound for $c_{0}$.

These examples may suffice to illustrate the use of the inequality (6). An arbitrary number of other necessary conditions for the nonoscillation of (1) can be obtained by different choices for the function $y(x)$ in (6).

3. A comparison theorem. Let $p(x)$ and $q(x)$ be continuous in an interval $[a, b]$, and consider the differential equations 


$$
\begin{aligned}
u^{\prime \prime}+p(x) u & =0, \\
v^{\prime \prime}+q(x) v & =0 .
\end{aligned}
$$

According to the Sturm comparison theorem all solutions of (15) must change their sign in $(a, b)$ if $u(a)=u(b)=0$ and $p(x) \leqq q(x)$ (but not $p(x) \equiv q(x)$ ) throughout the interval. The theorem of this section is based on the comparison of the integrals of the functions $p(x)$ and $q(x)$, rather than that of the functions themselves. We shall prove the following two different versions of this result.

THEOREM V. Let $p(x)$ be non-negative and continuous and let $q(x)$ be continuous in $[a, b]$. Further, let

$$
\int_{a}^{x} p(t) d t \leqq \int_{a}^{x} q(t) d t
$$

for $a<x<b$. If $\lambda$ and $\mu$ denote, respectively, the lowest eigenvalues of the differential systems

$$
\begin{array}{ll}
\phi^{\prime \prime}+\lambda p(x) \phi=0, & \phi^{\prime}(a)=\phi(b)=0 \\
\psi^{\prime \prime}+\mu q(x) \psi=0, & \psi^{\prime}(a)=\psi(b)=0
\end{array}
$$

then

$$
\mu \leqq \lambda
$$

where equality is excluded unless $p(x)$ and $q(x)$ coincide.

Theorem Va. Let $p(x), q(x)(p(x) \not \equiv q(x))$ be continuous and $p(x) \geqq 0$ in $[a, b]$, and let the equation (14) have a solution $u(x)$ for which $u^{\prime}(a)=u(b)=0$. If (16) holds for $a<x<b$ and $v(x)$ is a solution of (15) for which $v(a)>0$, $v^{\prime}(a) \leqq 0$, then at least one of the functions $v(x), v^{\prime}(x)$ must change its sign in $a<x<b$.

To prove Theorem $\mathrm{V}$, we multiply the equation (17) by $\phi$ and integrate from $a$ to $b$. We obtain

$$
\begin{aligned}
\int_{a}^{b} \phi^{\prime 2} d x & =-\int_{a}^{b} \phi \phi^{\prime \prime} d x=\lambda \int_{a}^{b} p \phi^{2} d x=\lambda \int_{a}^{b}\left[\int_{a}^{x} p(t) d t\right]^{\prime} \phi^{2} d x \\
& =\lambda\left[\phi^{2}(x) \int_{a}^{x} p(t) d t\right]_{a}^{b}-\lambda \int_{a}^{b}\left[\int_{a}^{x} p(t) d t\right]\left(\phi^{2}\right)^{\prime} d x \\
& =-\lambda \int_{a}^{b}\left[\int_{a}^{x} p(t) d t\right]\left(\phi^{2}\right)^{\prime} d x .
\end{aligned}
$$

Since $\lambda$ is the lowest eigenvalue of the system (17), $\phi(x)$ does not vanish in $a<x<b$, and we may therefore assume that $\phi(x) \geqq 0$. In view of (17) and the fact that $p(x) \geqq 0$, it follows therefore that $\phi^{\prime \prime}(x) \leqq 0$. Because of $\phi^{\prime}(a)=0$, we 
may thus conclude that $\phi^{\prime}(x) \leqq 0$ throughout the interval. Combining this with (16), we find that

$$
\begin{aligned}
-\int_{a}^{b}\left[\int_{a}^{x} p(t) d t\right]\left(\phi^{2}\right)^{\prime} d x & \leqq-\int_{a}^{b}\left[\int_{a}^{x} q(t) d t\right]\left(\phi^{2}\right)^{\prime} d x \\
& =\left[\phi^{2}(x) \int_{a}^{x} q(t) d t\right]_{a}^{b}+\int_{a}^{b} q(x) \phi^{2} d x=\int_{a}^{b} q(x) \phi^{2} d x .
\end{aligned}
$$

Hence

$$
\int_{a}^{b} \phi^{\prime 2} d x \leqq \lambda \int_{a}^{b} q(x) \phi^{2} d x .
$$

On the other hand, it follows from the minimum property of the lowest eigenvalue of the system (18) that

$$
\mu \int_{a}^{b} q(x) \phi^{2} d x<\int_{a}^{b} \phi^{\prime 2} d x \quad(p(x) \not \equiv q(x)) .
$$

We thus find that $\mu<\lambda$, which is the assertion of Theorem $V$.

Theorem Va would be an immediate corollary of Theorem V if we had the additional hypothesis $q(x) \geqq 0$. In order to avoid this assumption, we use a slightly different argument. From (14) and (15), we obtain

$$
\begin{aligned}
v(b) u^{\prime}(b)+u(a) v^{\prime}(a) & =\left[v u^{\prime}-u v^{\prime}\right]_{a}^{b}=\int_{a}^{b}\left(v u^{\prime \prime}-v u^{\prime \prime}\right) d x=\int_{a}^{b}(q-p) u v d x \\
& =\int_{a}^{b}\left[\int_{a}^{x}(q(t)-p(t) d t]^{\prime} u v d x .\right.
\end{aligned}
$$

Integrating by parts and noting that the integrated part vanishes, we find that

$$
v(b) u^{\prime}(b)+u(a) v^{\prime}(a)=\int_{a}^{b}\left[\int_{a}^{x}(p(t)-q(t)) d t\right]\left(v u^{\prime}+u v^{\prime}\right) d x .
$$

We now assume that neither $v(x)$ nor $v^{\prime}(x)$ change their signs in $a<x<b$. Because of $v(a)>0, v^{\prime}(a) \leqq 0$, we will then have $v(x) \geqq 0, v^{\prime}(x) \leqq 0$. As shown above, we have also $u^{\prime}(x)<0$ if $u(x)$ is non-negative in $a<x<b$. This may be assumed to be the case, since otherwise we could replace $b$ by the first zero of $u(x)$ at the right side of $a$. Utilizing (16), we thus find that $v(b) u^{\prime}(b)$ $+u(a) v^{\prime}(a)>0$ unless $p(x)$ and $q(x)$ coincide. But this is absurd, since $v(b)>0$, $u(a)>0, u^{\prime}(b)<0, v^{\prime}(a)<0$. At least one of the functions $v(x), v^{\prime}(x)$ must therefore change its sign in $(a, b)$, and Theorem $\mathrm{Va}$ is proved.

To illustrate the application of Theorem $\mathrm{V}$, set $q(x)=p(x)+\alpha\left(1-3 x^{2}\right)$, $a=0, b=1, \alpha>0, p(x)=p(-x)$. Since 


$$
\int_{0}^{x} q(t) d t=\int_{0}^{x} p(t) d t+\alpha x\left(1-x^{2}\right),
$$

(16) holds, and we have $\mu<\lambda$, where $\lambda$ and $\mu$ are, respectively, the lowest eigenvalues of the systems

$$
\begin{aligned}
u^{\prime \prime}+\lambda p(x) u & =0, & u( \pm 1) & =0, \\
v^{\prime \prime}+\mu\left[p(x)+\alpha\left(1-3 x^{2}\right)\right] v & =0, & (v \pm 1) & =0 .
\end{aligned}
$$

In particular, if we set $p(x)=x^{2}, \alpha=1 / 3$, we have $q(x)=1 / 3$. Hence, $\mu=3 \pi^{2} / 4$ and we obtain $\lambda>3 / 4 \pi^{2}$, where $\lambda$ is the lowest eigenvalue of $u^{\prime \prime}+\lambda x^{2} u=0$, $u( \pm 1)=0$. This is considerably better then the estimate $\lambda>\pi^{2} / 4$ obtained from $x^{2} \leqq 1$ and the Sturm comparison theorem.

4. A general oscillation criterion. We recall from $\$ 1$ the definition of the oscillation constant $\mu$ of the equation

$$
u^{\prime \prime}+q(x) u=0,
$$$$
q(x) \geqq 0,
$$

where $q(x)$ is continuous for $x>0 . \mu$ is defined by the condition that

$$
u^{\prime \prime}+\lambda q(x) u=0
$$

is oscillatory for $\lambda>\mu$ and nonoscillatory for $\lambda<\mu$. We shall also refer to $\mu$ as the "oscillation constant of the function $q(x)$ ". As pointed out in $\S 1$, the cases in which $\mu=\infty$ or $\mu=0$-the strongly oscillatory and the strongly nonoscillatory case - can be completely characterized by the conditions given in Theorem II. We may therefore restrict ourselves to equations with finite positive $\mu$, i.e., conditionally oscillatory equations.

In the following theorem, the oscillatory behavior of the equation (1) is compared with that of a conditionally oscillatory equation whose oscillation constant is known.

THEOREM VI. Let $p(x)$ and $q(x)$ be continuous and non-negative for $0 \leqq x<\infty$ and denote by $\mu(0<\mu<\infty)$ the oscillation constant of $q(x)$. If $A$ and $B$ are defined by

$$
A=\limsup _{x \rightarrow \infty}\left(\int_{x}^{\infty} p(t) d t / \int_{x}^{\infty} q(t) d t\right)
$$

and

$$
B=\liminf _{x \rightarrow \infty}\left(\int_{x}^{\infty} p(t) d t / \int_{x}^{\infty} q(t) d t\right),
$$

then the equation (1) will be oscillatory if $B>\mu$ and nonoscillatory if $A<\mu$.

Since $A=B=1$ if $p(x) \equiv q(x)$, both bounds are evidently sharp. If $q(x)$ $\equiv x^{-2}$, then $\mu=1 / 4, \int_{x}^{\infty} q(t) d t=x^{-1}, A=g^{*}, B=g_{*}$ (as defined in (2) and (3)), and Theorem VI reduces to the result of Hille [4] quoted further above. We 
remark that Theorem VI may, in turn, be deduced from Hille's Theorem VII the proof of which is based on the consideration of a certain nonlinear integral equation. The direct proof given here employs linear methods.

Suppose (1) is nonoscillatory. There exists a positive $a$ such that (1) is nonoscillatory in $(a, \infty)$ and, as shown in $\$ 2$ (formula (6)), we have

$$
\int_{a}^{b} p(x) w^{2} d x<\int_{a}^{b} w^{\prime 2} d x
$$

where $w(a)=0, w(x) \in D^{1}$ in $(a, \infty)$ and $w(x)$ is otherwise arbitrary. With the notation

$$
\sigma(x)=\left(\int_{x}^{\infty} p(t) d t / \int_{x}^{\infty} q(t) d t\right)
$$

we have

$$
\begin{aligned}
\int_{a}^{b} p(x) w^{2} d x & =-\int_{a}^{b}\left[\sigma(x) \int_{x}^{\infty} q(t) d t\right]^{\prime} w^{2} d x \\
& =-\sigma(b) w^{2}(b) \int_{b}^{\infty} q(t) d t+\int_{a}^{b} \sigma(x)\left[\int_{x}^{\infty} q(t) d t\right]\left(w^{2}\right)^{\prime} d x .
\end{aligned}
$$

If $a$ is taken large enough, we will have $\sigma(x) \geqq B-\epsilon$, where $B$ is defined in (22) and $\epsilon$ is positive and arbitrarily small. If $w(x)$ is a function which increases with $x$, it follows therefore that

$$
\begin{aligned}
\int_{a}^{b} p(x) w^{2} d x & \geqq-\sigma(b) w^{2}(b) \int_{a}^{\infty} q(t) d t+(B-\epsilon) \int_{a}^{b}\left[\int_{a}^{\infty} q(t) d t\right]\left(w^{2}\right)^{\prime} d x \\
& =[B-\sigma(b)-\epsilon] w^{2}(b) \int_{b}^{\infty} q(t) d t+\int_{a}^{b} q(x) w^{2} d x .
\end{aligned}
$$

This relation holds for any $b$ which is larger than $a$. As (22) shows, there exist arbitrarily large values of $b$ for which $\sigma(b) \leqq B+\epsilon$. For such $b$, we obtain

$$
\int_{a}^{b} p(x) w^{2} d x \geqq-2 \epsilon w^{2}(b) \int_{b}^{\infty} q(t) d t+(B-\epsilon) \int_{a}^{b} q(x) w^{2} d x .
$$

We now identify $w(x)$ with the first eigenfunction of the system

$$
w^{\prime \prime}+\nu q(x) w=0, \quad w(a)=w^{\prime}(b)=0 .
$$

We then have $w^{\prime}(x) \geqq 0(a \leqq x \leqq b)$,

$$
\nu \int_{a}^{b} q(x) w^{2} d x=\int_{a}^{b} w^{\prime 2} d x
$$

and 


$$
w^{2}(b)=\left(\int_{a}^{b} w^{\prime} d x\right)^{2} \leqq(b-a) \int_{a}^{b} w^{\prime 2} d x
$$

whence

$$
\int_{a}^{b} p(x) w^{2} \geqq-2 \epsilon(b-a) \int_{b}^{\infty} q(t) d t \int_{a}^{b} w^{\prime 2} d x+\frac{B-\epsilon}{\nu} \int_{a}^{b} w^{\prime 2} d x .
$$

Combining this with (23), we are led to the inequality

$$
1 \geqq-2 \epsilon(b-a) \int_{b}^{\infty} q(t) d t+\frac{B-\epsilon}{\nu} .
$$

If $\lambda=\mu-\epsilon$, the equation (20) is nonoscillatory. In view of (8), we thus have

$$
(\mu-\epsilon)(b-a) \int_{b}^{\infty} q(t) d t \leqq 1
$$

and therefore

$$
1 \geqq-\frac{2 \epsilon}{\mu-\epsilon}+\frac{B-\epsilon}{\nu} .
$$

For $\lambda=\mu+\epsilon$, the equation (20) is oscillatory. Writing the system (25 in the form

$$
w^{\prime \prime}+\left(\frac{\nu}{\mu+\epsilon}\right)(\mu+\epsilon) q(x) w=0, \quad w(a)=w^{\prime}(b)=0,
$$

we may therefore conclude from Theorem I that $\nu(\mu+\epsilon)^{-1} \leqq 1$ if $b$ is taken large enough. It follows that

$$
1 \geqq-\frac{2 \epsilon}{\mu-\epsilon}+\frac{B-\epsilon}{\mu+\epsilon} .
$$

Since $\epsilon$ may be taken arbitrarily small, this shows that, for a nonoscillatory equation, the quantity $B$ defined in (22) cannot exceed $\mu$. This establishes the first part of Theorem VI.

The truth of the second half of our assertion becomes apparent if we express our result in terms of the oscillation constant $\mu_{0}$ of the function $p(x)$. By the definition of $\mu_{0}$, the equation $u^{\prime \prime}+\left(\mu_{0}-\delta\right) p(x) u=0$ will be nonoscillatory if $\delta$ is an arbitrarily small positive constant. The preceding argument will therefore remain valid if the quantity $B$ is replaced by $\left(\mu_{0}-\delta\right) B$, and we may reformulate our result in the following manner.

THEOREM VIa. Let $p(x)$ and $q(x)$ be continuous and non-negative in $(0, \infty)$ and let $\mu_{0}$ and $\mu$ denote the oscillation constants of $p(x)$ and $q(x)$, respectively. If $\mu_{0} \neq 0, \infty, \mu \neq 0, \infty$, and $B$ is defined by (22), then 


$$
\mu_{0} B \leqq \mu .
$$

To establish the second half of the assertion of Theorem VI, we exchange the roles of $p(x)$ and $q(x)$. Since, in view of (21),

$$
\lim _{x \rightarrow \infty} \inf \left(\int_{x}^{\infty} q(x) d x / \int_{x}^{\infty} p(x) d x\right)=\frac{1}{A},
$$

(26) yields the inequality $\mu \leqq \mu_{0} A$. If (1) is oscillatory, we must have $\mu_{0} \leqq 1$ and, therefore, $\mu \leqq \mu_{0} A \leqq A$. This completes the proof of Theorem VI.

5. Asymptotic estimates for the number of zeros. If the equation (1) is oscillatory and

$$
\left(\frac{1}{(p(x))^{1 / 2}}\right)^{\prime} \rightarrow 0
$$

as $x \rightarrow \infty$, and $N[a, b]$ denotes the number of zeros of a solution $y(x)$ of $(1)$ in the interval $(a, b)$ then, as shown by Wiman [10] (for a different proof, see $[3])$,

$$
N[a, b] \sim \frac{1}{\pi} \int_{a}^{b}(p(x))^{1 / 2} d x
$$

where the symbol $\sim$ means that the ratio of the two quantities tends to 1 as $b \rightarrow \infty$. In the present section we shall develop a procedure for the derivation of more general estimates of this type. Again, our treatment will be based on the eigenvalue problem for the equation $\left(4^{\prime}\right)$ with suitable boundary conditions. Our basic result is stated in the following

LEMmA. Let $a=a_{0}, a_{1}, \cdots, a_{n}=b\left(a_{0}<\cdots<a_{n}\right)$ be consecutive zeros of a solution of (1) and let $\lambda=\lambda_{n}$ be the nth eigenvalue of the system

$$
u^{\prime \prime}(t)+\lambda q(t) u(t)=0, \quad u(0)=u(T)=0,
$$

where $q(t)$ is non-negative and continuous in $[0, \infty)$. If $t=t(x)$ and $T$ are defined by

$$
\begin{aligned}
& \int_{0}^{t}(q(s))^{1 / 2} d s=\int_{a}^{x}(p(\zeta))^{1 / 2} d \zeta \\
& \int_{0}^{T}(q(s))^{1 / 2} d s=\int_{a}^{b}(p(\zeta))^{1 / 2} d \zeta
\end{aligned}
$$

and we have

$$
\left|\frac{d}{d x}\left[\frac{1}{(p(x))^{1 / 2}}\right]-\frac{d}{d t}\left[\frac{1}{(q(t))^{1 / 2}}\right]\right| \leqq M \quad \text { for } a \leqq x \leqq b
$$


then

$$
\left|\left(\lambda_{n}\right)^{1 / 2}-1\right| \leqq \frac{1}{2} M
$$

This result has the character of a comparison theorem. To obtain from (33) a useful estimate for the number of zeros it is, of course, necessary to solve the eigenvalue problem (29), and there are not many cases in which this can be done explicitly. On the other hand, each function $q(t)$ for which the problem (29) can be solved leads to an estimate for the number of zeros of the class of oscillatory equations (1) for which (32) holds. For the sub-class of equations for which $M \rightarrow 0$ for $a \rightarrow \infty$, (33) will yield an asymptotic formula for the number of zeros.

To prove the lemma, we consider the Rayleigh quotient

$$
J=J(y)=\int_{a}^{b} y^{\prime 2} d x / \int_{a}^{b} p(x) y^{2} d x,
$$

where $y(x) \in C^{\mathbf{l}}$ in $[a, b]$. Writing $y=u \phi$ and introducing a new variable $t=t(x)$, we obtain

$$
\begin{aligned}
J & =\frac{\int_{a}^{b}\left(\phi u^{\prime}+u \phi^{\prime}\right)^{2} d x}{\int_{a}^{b} p \phi^{2} u^{2} d x} \\
& =\frac{\int_{0}^{T}\left[\phi\left(\frac{d t}{d x}\right)^{1 / 2} \frac{d u}{d t}+u \phi^{\prime}\left(\frac{d x}{d t}\right)^{1 / 2}\right]^{2} d t}{\int_{0}^{T} p \phi^{2} \frac{d x}{d t} u^{2} d t},
\end{aligned}
$$

where $t(a)=0, t(b)=T, u(t)=v(x)$, and $\phi^{\prime}=d \phi / d x$. We now identify $t=t(x)$ with the transformation given by (30) and we set $\phi^{2}=d x / d t(\phi>0)$. An elementary computation shows that

$$
\frac{2}{(q(t))^{1 / 2}} \frac{d \phi}{d x} \frac{d x}{d t}=\frac{d}{d x}\left[\frac{1}{(p(x))^{1 / 2}}\right]-\frac{d}{d t}\left[\frac{1}{(q(t))^{1 / 2}}\right] .
$$

We thus have the identity

$$
J=\frac{\int_{0}^{T}\left(u^{\prime}+\frac{q^{1 / 2}}{2}\left[\left(\frac{1}{p^{1 / 2}}\right)^{\prime}-\left(\frac{1}{q^{1 / 2}}\right)^{\prime}\right] u\right)^{2} d t}{\int_{0}^{T} q u^{2} d t}
$$


where primes denote differentiation with respect to the variable on which the function depends explicitly.

Using (32) and the Schwarz inequality we obtain

$$
\begin{aligned}
J & \leqq \int_{0}^{T} u^{\prime 2} d t / \int_{0}^{T} q u^{2} d t+M\left(\int_{0}^{T} q^{1 / 2}\left|u u^{\prime}\right| d t / \int_{0}^{T} q u^{2} d t\right)+\frac{1}{4} M^{2} \\
& \leqq \int_{0}^{T} u^{\prime 2} d t / \int_{0}^{T} q u^{2} d t+M\left(\int_{0}^{T} u^{\prime 2} d t / \int_{0}^{T} q u^{2} d t\right)^{1 / 2}+\frac{1}{4} M^{2} \\
& =\left(\left(\int_{0}^{T} u^{\prime 2} d t / \int_{0}^{T} q u^{2} d t\right)^{1 / 2}+\frac{M}{2}\right)^{2},
\end{aligned}
$$

or, with the notation

$$
\begin{array}{r}
J_{1}=J_{1}(u)=\int_{0}^{T} u^{\prime 2} d t / \int_{0}^{T} q u^{2} d t, \\
(J(y))^{1 / 2} \leqq\left(J_{1}(u)\right)^{1 / 2}+M / 2 .
\end{array}
$$

To show that we have also

$$
(J(y))^{1 / 2} \geqq\left(J_{1}(u)\right)^{1 / 2}-M / 2,
$$

and thus

$$
\left|(J(y))^{1 / 2}-\left(J_{1}(u)\right)^{1 / 2}\right| \leqq M / 2,
$$

we remark that we may assume $\left(J_{1}\right)^{1 / 2}>M / 2$, since otherwise the relation holds trivially. We then have

$$
0 \leqq\left(J_{1}\right)^{1 / 2}-\frac{1}{2} M \leqq\left(J_{1}\right)^{1 / 2}-\left(\int_{0}^{T} \phi^{2} q u^{2} d t / \int_{0}^{T} q u^{2} d t\right)^{1 / 2},
$$

where $\phi$ denotes the quantity estimated in (32). Hence

$$
\begin{aligned}
&\left(\left(J_{1}\right)^{1 / 2}-\frac{1}{2} M\right)^{2} \\
& \quad \leqq J_{1}-2\left(J_{1}\right)^{1 / 2}\left(\int_{0}^{T} \phi^{2} q u^{2} d t / \int_{0}^{T} q u^{2} d t\right)^{1 / 2}+\int_{0}^{T} \phi^{2} q u^{2} d t / \int_{0}^{T} q u^{2} d t \\
& \quad \leqq J_{1}-2 \int_{0}^{T} q^{1 / 2}\left|\phi u u^{\prime}\right| d t / \int_{0}^{T} q u^{2} d t+\int_{0}^{T} \phi^{2} q u^{2} d t / \int_{0}^{T} q u^{2} d t \\
& \quad=\int_{0}^{T}\left(\left|u^{\prime}\right|-q^{1 / 2}|u \phi|\right)^{2} d t / \int_{0}^{T} q u^{2} d t \\
& \leqq \int_{0}^{T}\left(u^{\prime}+q^{1 / 2} \phi u\right)^{2} d t / \int_{0}^{T} q u^{2} d t .
\end{aligned}
$$


In view of (35) and the definition of $\phi$, we thus find that $\left(J_{1}\right)^{1 / 2} \leqq J^{1 / 2}+M / 2$. Together with (37), this proves the inequality (38).

According to a well-known result of Courant [1], the $n$th eigenvalue of the problem (29) is given by

$$
\lambda=\sup \lambda\left(g_{1}, \cdots, g_{n-1}\right),
$$

where

$$
\lambda\left(g_{1}, \cdots, g_{n-1}\right)=\inf J_{1}(w),
$$

$w(t) \in C^{1}[0, T], g_{\nu} \in C^{1}[0, T](\nu=1, \cdots, n-1), w(0)=w(t)=g_{\nu}(0)=g_{\nu}(T)=0$, $J_{1}(w)$ is defined by (36), and

$$
\int_{0}^{T} w(t) g_{\nu}(t) d t=0, \quad \nu=1, \cdots, n-1 .
$$

In (40), the $g_{1}, \cdots, g_{n-1}$ are given and $w$ ranges over all admissible functions which satisfy (41), while in (39) the $g_{1}, \cdots, g_{n-1}$ range over all possible sets of $n-1$ admissible functions.

Similarly, the $n$th eigenvalue of the system

$$
\psi^{\prime \prime}+\Lambda p(x) \psi=0, \quad \psi(a)=\psi(b)=0
$$

is given by $\Lambda_{n}=\sup \Lambda\left(h_{1}, \cdots, h_{n-1}\right)$, where

$$
\begin{aligned}
\Lambda\left(h_{1}, \cdots, h_{n-1}\right) & =\inf J(\phi), \\
\int_{a}^{b} \phi(x) & h_{\nu}(x) d x=0, \quad \nu=1, \cdots, n-1,
\end{aligned}
$$

$\phi(x), h_{1}(x), \cdots, h_{n-1}(x)$ are admissible functions and $J(\phi)$ is defined by (34).

(43) may be written in the form

$$
\int_{0}^{T} \phi[x(t)]\left[h_{\nu}[x(t)] \frac{d x}{d t}\right] d t=0, \quad \nu=1, \cdots, n-1,
$$

where the variables $x$ and $t$ are related by means of (30), and (41) may be replaced by

$$
\int_{a}^{b} w[t(x)]\left[g_{\nu}[t(x)] \frac{d t}{d x}\right] d x=0, \quad \nu=1, \cdots, n-1 .
$$

Since the transformation $x \rightarrow t$ carries admissible functions in $a \leqq x \leqq b$ into admissible functions in $0 \leqq t \leqq T$ and vice versa, this shows that there exists a $(1,1)$ correspondence between the totality of functions $g_{\nu}$ which are used in the side conditions (41), and the class of functions $h_{\nu}$ used in (43). The same is true of the class of functions $w$ and the class $\phi$, and it follows therefore from (38) and a standard argument [1] that 


$$
\left|\left(\Lambda_{n}\right)^{1 / 2}-\left(\lambda_{n}\right)^{1 / 2}\right| \leqq M / 2 .
$$

By a classical result, the $n$th eigenfunction of the system (42) has precisely $n+1$ zeros in $[a, b]$, including those at $x=a$ and $x=b$. By our assumption, the same is true of one of the solutions of (1). Since there cannot exist two different values of $\Lambda$ for which (42) has a solution with these properties, we must have $\Lambda_{n}=1$. In view of (44), this completes the proof of the lemma.

As stated above, the usefulness of the lemma depends on our ability to find the eigenvalues of the system (29). The two simplest cases are those in which $q(t) \equiv 1$ and $q(t)=\beta^{2}(t+1)^{-2}$. If $q(t) \equiv 1$, (29) reduces to $u^{\prime \prime}+\lambda u=0, u(0)$ $=u(T)=0$, and the corresponding eigenvalues are

$$
\lambda_{n}=\left(\frac{\pi n}{T}\right)^{2}=\left(\frac{\pi n}{\int_{a}^{b}(p(x))^{1 / 2} d x}\right)^{2},
$$

where (31) has been used. Since $\left(q^{-1 / 2}\right)^{\prime}=0$, an application of the lemma will thus give the following result

Theorem VII. Let $p(x)>0, p(x) \in C^{\prime}(0, \infty)$, and let $a_{0}=a, a_{1}, \cdots, a_{n}=b$ $\left(0<a_{0}<a_{1}, \cdots,<a_{n}\right)$ be $n+1$ consecutive zeros of a solution of (1). If

$$
\left|\left(\frac{1}{(p(x))^{1 / 2}}\right)^{\prime}\right| \leqq 2 R, \quad a \leqq x \leqq b,
$$

then

$$
\frac{1-R}{\pi} \int_{a}^{b}(p(x))^{1 / 2} d x \leqq n \leqq \frac{1+R}{\pi} \int_{a}^{b}(p(x))^{1 / 2} d x .
$$

This contains the above-mentioned result by Wiman as a special case. Indeed, if $\left(p^{-1 / 2}\right)^{\prime} \rightarrow 0$, we may take $R=\epsilon$, where $\epsilon$ is arbitrarily small for $x \geqq c$, provided $c$ is taken large enough. Taking $b>c$ and letting $b \rightarrow \infty$ for fixed $c$ we obtain (28), provided $(p(x))^{1 / 2}$ is not integrable in $(a, \infty)$. This is indeed the case since, as (46) shows, (1) is nonoscillatory if $\int_{a}^{\infty} p^{1 / 2} d x<\infty$ and the condition (45) holds with a finite $R$.

In the second example we set $g(t)=\beta^{2}(t+1)^{-2}(\beta>2)$. By (31), we have

$$
\beta \log (T+1)=\int_{a}^{b}(p(x))^{1 / 2} d x,
$$

and the eigenvalues of (29) are

$$
\lambda_{n}=\frac{1}{4 \beta^{2}}+\left(\frac{\pi n}{\beta \log (T+1)}\right)^{2}=\frac{1}{4 \beta^{2}}+\left(\frac{\pi n}{\int_{a}^{b}(p(x))^{1 / 2} d x}\right)^{2} .
$$


Since $\left(q^{-1 / 2}\right)^{\prime}=\beta^{-1}$, this proves the following result.

TheOREM VIIa. Let $n, p(x), a, b$ have the same meaning as in Theorem VII. If

$$
\left|\left(\frac{1}{(p(x))^{1 / 2}}\right)^{\prime}-2 \alpha\right| \leqq 2 R \quad 0<\alpha<1, a \leqq x \leqq v
$$

then

$$
\begin{aligned}
\left((1-R)^{2}-\alpha^{2}\right)\left(\frac{1}{\pi} \int_{a}^{b}(p(x))^{1 / 2} d x\right)^{2} & \\
& \leqq n^{2} \leqq\left((1+R)^{2}-\alpha^{2}\right)\left(\frac{1}{\pi} \int_{a}^{b}(p(x))^{1 / 2} d x\right)^{2} .
\end{aligned}
$$

If $\left(p^{-1 / 2}\right)^{\prime} \rightarrow 2 \alpha$, this reduces to the asymptotic formula

$$
n \sim \frac{\left(1-\alpha^{2}\right)^{1 / 2}}{\pi} \int_{a}^{b}(p(x))^{1 / 2} d x
$$

which can also be obtained by applying Wiman's formula (28) to a suitably transformed equation (1) [9].

\section{REFERENCES}

1. R. Courant and D. Hilbert, Methods of mathematical physics, New York, Interscience, 1953.

2. W. B. Fite, Concerning the zeros of the solutions of certain differential equations, Trans. Amer. Math. Soc. vol. 19 (1917) pp. 341-352.

3. P. Hartman and A. Wintner, The asymptotic arcus variation of solutions of real linear differential equations of second order, Amer. J. Math. vol. 70 (1948) pp. 1-10.

4. E. Hille, Non-oscillation theorems, Trans. Amer. Math. Soc. vol. 64 (1948) pp. 234-252.

5. A. Kneser, Untersuchungen über die reellen Nullstellen der Integrale linearer Differentialgleichungen, Math. Ann. vol. 42 (1893) pp. 409-435.

6. W. Leighton, The detection of the oscillation of solutions of a second order linear differential equation, Duke Math. J. vol. 17 (1950) pp. 57-62.

7. - On self-adjoint differential equations of second order, J. London Math. Soc. vol. 27 (1952) pp. 37-47.

8. R. A. Moore, The behavior of solutions of a linear differential equation of second order, Pacific Journal of Mathematics vol. 5 (1955) pp. 125-145.

9. R. L. Potter, On self-adjoint differential equations of second order, Pacific Journal of Mathematics vol. 3 (1953) pp. 467-491.

10. A. Wiman, Über die reellen Lösungen der linearen Differentialgleichungen zweiter Ordnung, Arkiv för Matematik Astronomi och Fysik vol. 12 No. 14 (1917).

11. A. Wintner, On the Laplace-Fourier transcendents occurring in mathematical physics, Amer. J. Math. vol. 69 (1947) pp. 87-98.

12. - A criterion of oscillatory stability, Quarterly of Applied Mathematics vol. 7 (1949) pp. 115-117.

Carnegie Institute of Technology, Pittsburgh, Pa. 